Topiques, études satoriennes

Topoï Studies, Journal of the SATOR

\title{
Nourritures terrestres, nourritures célestes et rhétorique du sublime dans l'art de Bossuet panégyriste
}

\section{Nicolas Pelleton}

Volume 5, 2021

Le manger et le boire dans la fiction narrative

URI: https://id.erudit.org/iderudit/1081526ar

DOI: https://doi.org/10.7202/1081526ar

See table of contents

Publisher(s)

SATOR, Société d'Analyse de la Topique Romanesque d'Ancien Régime

ISSN

2369-4831 (digital)

Explore this journal

Cite this article

Pelleton, N. (2021). Nourritures terrestres, nourritures célestes et rhétorique du sublime dans l'art de Bossuet panégyriste. Topiques, études satoriennes / Topoï Studies, Journal of the SATOR, 5, 105-121. https://doi.org/10.7202/1081526ar
Article abstract

In the Panegyrics of saint Gorgon, Bossuet uses the topics of eating and drinking, and changes its meaning under the paradigm of catholic dogma : it doesn't help any more to express humiliation, but to express the greatness of christianism. This paradoxical construction of christian sublime forms the bedrock of an approach of language as eating and digesting : the topical construction raises the legendary aspect of the narrative of martyrdom - a border case of narrative fiction -, which is itself a component of the poetics of panegyric.
This document is protected by copyright law. Use of the services of Érudit (including reproduction) is subject to its terms and conditions, which can be viewed online.

https://apropos.erudit.org/en/users/policy-on-use/ 


\section{Nourritures terrestres, nourritures célestes et rhétorique du sublime dans l'art de Bossuet panégyriste}

Nicolas Pelleton

CELIS, Université Clermont Auvergne

Selon André Jolles, le XVII siècle est une « époque importante [...] pour la définition de la sainteté ${ }^{\prime} »$. En effet, c'est à cette époque que 1'Église catholique, consciente de ses pouvoirs institutionnels et moraux, met en forme de façon canonique la vie des saints et des saintes qui ont souffert pour la plus grande gloire du Christ. L'Église se veut alors « l'institution qui proclame la sainteté ${ }^{2} »$, par des procédures juridiques qui cherchent à établir les vertus théologales et morales des saints ${ }^{3}$, afin de les offrir en exemple aux fidèles. Aussi la forme du panégyrique, genre oratoire chrétien qui consiste en l'éloge d'un saint ou d'une sainte à des fins d'édification des fidèles, a-t-elle été pratiquée au Grand Siècle par Bossuet. L'évêque de Meaux est conscient du caractère grandiose de la sainteté : «Apprenez de là, ô peuple religieux, combien est honorable et précieuse devant Dieu [...] la mort de ses saints ${ }^{4} \gg$. Du point de vue rhétorique, cet hommage à la sainteté s'actualise par le sublime.

Au XVII siècle, c'est le Traité du sublime de Longin, traduit du grec par Boileau, qui constitue la référence théorique. Longin définit son objet en ces termes :

Je veux dire, qu'il faut bien se donner de garde d'y prendre [dans les « ouvrages des poètes et des orateurs »] pour Sublime une certaine apparence de grandeur bâtie ordinairement sur de grands mots assemblés au hasard, et qui n'est, à la bien examiner, qu'une vaine enflure de paroles, plus digne en effet de mépris que d'admiration. Car tout ce qui est véritablement Sublime, a cela de propre, quand on l'écoute, qu'il élève l'âme, et lui fait concevoir une plus haute opinion d'elle-même, la remplissant de joie et de je ne sais quel noble orgueil, comme si c'était elle qui eût produit les choses qu'elle vient simplement d'entendres.

Longin identifie ici deux sources du sublime : le style et les pensées, qui doivent être dissociés de toute forme de vulgarité et d'abaissement. Le sublime suppose de susciter chez l'auditeur

\footnotetext{
${ }^{1}$ André Jolles, Formes simples, 1972, p. 28.

2 Ibid., p. 29.

${ }^{3}$ Ibid., p. 30.

${ }^{4}$ Bossuet, Pensées chrétiennes et morales, dans Bossuet, Euvres oratoires, 1926, t. 6, p. 704.

${ }^{5}$ Longin, Traité du sublime, 1995, p. 81.
} 
une élévation de l'âme, une joie, une noblesse. Le sublime implique une réaction psychologique chez l'auditeur. Il peut donc poursuivre un but édifiant, et se charger d'une valeur rhétorique.

Il semble alors paradoxal de parler du sublime des Panégyriques de saint Gorgon: non seulement ces discours s'appuient sur la topique alimentaire, traditionnellement symbole $\mathrm{d}^{\prime}$ abaissement ${ }^{6}$, mais cette topique est associée au cannibalisme. Pour autant, si un tel récit annule apparemment toute forme de sublime, il correspond à une double tension, propre à l'axiologie catholique.

Grâce à une approche topique ${ }^{7}$ et énonciative des deux Panégyriques de saint Gorgon, nous étudierons les recatégorisations axiologiques que Bossuet fait subir à la topique alimentaire. Ces recatégorisations sont le support d'une double tension, à la fois abaissante et glorificatrice, par laquelle le prédicateur met en place un sublime au service de l'ambition morale et édifiante du panégyrique. C'est pourquoi il nous faudra réfléchir aux liens entre la construction légendaire du récit du martyre, et la poétique du panégyrique : la première appartient pleinement à la seconde, et nourrit la rhétorique du sublime.

\section{(S')alimenter : les modalités narratives de la topique alimentaire}

C'est le 9 septembre $1649^{8}$ que le jeune abbé Bossuet, sous-diacre de la cathédrale de Metz $^{9}$, prononce un Premier Panégyrique de saint Gorgon, qui est le saint patron de la ville. Il se livrera également à cet exercice en 1654 , avec un autre panégyrique. C'est aussi à cette époque que Bossuet commence à poser les fondements de son autorité.

Saint Gorgon est le saint patron de Metz, depuis qu'en « [1]'an du Seigneur 763, un évêque de Metz, neveu du roi Pépin, en fit la translation [celle du corps de saint Gorgon] dans les Gaules et le déposa au monastère de Gorze ${ }^{10}$ ». La Légende dorée lie la figure de Gorgon à celle de Dorothée, tous deux favoris « de Dioclétien à Nicomédie ${ }^{11}$ ». Ils « renoncèrent aux dignités dont ils jouissaient depuis longtemps, afin de suivre leur roi avec plus de liberté et se

\footnotetext{
${ }^{6}$ Envisagé comme « principe artistique », le « rabaissement » propre aux motifs du « bas corporel » suppose que « toutes les choses sacrées et élevées [...] sont réinterprétées sur le plan matériel et corporel » (Mikhaïl Bakhtine, L'Euvre de François Rabelais et la culture populaire au Moyen Âge et sous la Renaissance, 1970, p. 368).

${ }^{7}$ Rappelons qu'un des objectifs de la SATOR est de « réévalu[er] [...] l'histoire littéraire, à partir d'une meilleure connaissance des structures, formes et thèmes narratifs ».

(https://satorbase.org/index.php?do=outils\#objectifs, page consultée le 12/05/2019).

${ }^{8}$ Les années 1648 et 1649 représentent « un tournant décisif dans la vie de Bossuet », parce que « [c]'est l'époque du choix définitif de la vie ecclésiastique [...] » (Georges Minois, Bossuet. Entre Dieu et le Soleil, 2003, p. 60).

${ }^{9}$ Notice introductive au Premier Panégyrique de saint Gorgon, dans Bossuet, Euvres oratoires, 1926, t. 1, p. 31.

${ }^{10}$ Jacques de Voragine, La Légende dorée, 1967, t. 2, p. 188.

${ }^{11}$ Ibid.
} 
déclarèrent ouvertement chrétiens ${ }^{12}$ ». L'empereur « fut très chagrin » de « perdre des hommes de ce rang, nourris dans son palais et autant distingués par leur conduite que par la noblesse de leur naissance ${ }^{13} \gg$. Notons ici que La Légende dorée associe la légende de saint Gorgon à la topique alimentaire, grâce au lexème «nourris », qui renvoie à la fois à la nourriture et à l'éducation. Les deux nouveaux convertis résistent aux " menaces » et aux «promesses » de «César ${ }^{14} »$; aussi la réaction de celui-ci devant l'inflexibilité de ses anciens favoris est-elle d'une violence extrême :

[...] on les fit étendre sur le chevalet, où après avoir été déchirés avec des fouets et des ongles de fer par tout le corps, ils furent couverts de vinaigre et de sel ; leurs entrailles étaient presque à nu. Et comme ils supportaient ces tourments avec grande joie, on les fit rôtir sur un gril, où il semblait qu'ils étaient couchés comme sur un lit de fleurs, sans éprouver la moindre souffrance. Enfin par l'ordre de César, on les pendit avec un lacet; leur corps furent jetés aux loups et aux chiens; mais ils furent recueillis intacts par les fidèles ${ }^{15}$.

Comme dans tout texte de nature hagiographique, le texte de La Légende dorée consacré à saint Gorgon suppose une trame narrative; cette configuration énonciative est constitutive de la forme même de la légende, qui « raconte la vie d['un] saint », qui « est une Vie $(v i t a)^{16} »$. Aussi la forme du panégyrique, qui trouve sa matière dans les documents hagiographiques, reprend-elle en partie cette forme narrative. Des origines du saint à son martyre, le but illocutoire du panégyrique consiste à glorifier la Passion du Christ à travers la mise en discours de « la vertu agissante ${ }^{17}{ }$ d'un saint. La dispositio du Premier Panégyrique propose un parcours à la fois théologique et chronologique. Dans le premier point, le prédicateur évoque les realia de la domus et de l'otium des empereurs :

Saint Gorgon vivait en la cour des empereurs Dioclétian et Maximian ${ }^{18}$, et avait une charge très considérable dans leur maison. [...] Surtout quiconque a tant soit peu lu l'histoire romaine y a pu remarquer quel crédit les empereurs donnaient ordinairement à leurs domestiques que leurs offices appelaient plus souvent près de leurs personnes ${ }^{19}$.

\footnotetext{
${ }^{12}$ Ibid.

${ }^{13} \mathrm{Ibid}$.

${ }^{14}$ Ibid.

${ }^{15} \mathrm{Ibid}$.

${ }^{16}$ André Jolles, Formes simples, op. cit., p. 38.

${ }^{17}$ Ibid.

${ }^{18}$ Les graphies « Dioclétian » et « Maximian » sont celles de Bossuet.

${ }^{19}$ Bossuet, Premier Panégyrique de saint Gorgon, dans Bossuet, Euvres oratoires, 1926, t. 1, p. 36. Nous renverrons désormais à ce discours grâce à l'abréviation $1 P d s G$.
} 
Dans le second point, la narration se propose d'évoquer la vie publique des empereurs, leur negotium :

Saint Gorgon ne l'a pas eu si aisé. Ce n'a pas été tout d'avoir méprisé les grandeurs ; l'empereur lui fit payer bien cher la grâce qu'il lui avait faite de le recevoir en son amitié. Outre la haine qu'il avait généralement pour tous les chrétiens [...], il était encore rongé d'un secret déplaisir d'avoir nourri en sa maison un ennemi de l'empire, et même de lui avoir donné part à sa confidence ${ }^{20}$.

Ici, la dispositio du discours n'associe pas la topique alimentaire aux realia de la vie «domestique » des empereurs, mais à l'évocation historique et politique de la haine des empereurs pour les chrétiens (grâce au participe passé « rongé »), c'est-à-dire, à un cadre narratif qui ne lui convient pas. Le Premier Panégyrique est donc construit sur une tension, fondée sur une recatégorisation paradigmatique de la topique alimentaire par le cadre narratif. Ce phénomène de recatégorisation suppose que l'utilisation narrative de la topique alimentaire est construite sur des effets de syllepse. Aussi est-ce le lexique qui fonde le paysage gustatif des deux discours, dont la valeur nettement dysphorique est apparemment éloignée de toute forme de sublime.

On note tout d'abord que manger n'est pas (se) nourrir. L'empereur qui condamne saint Gorgon est « rongé » de haine envers les chrétiens. Le Christ, imité par saint Gorgon, dit être « dévor[é] » par « une soif ardente ${ }^{21}$ ». Dans l'un et l'autre de ces exemples, les lexèmes « rongé » et « dévorait » sont employés par syllepse. Bossuet les utilise donc dans un but énonciatif précis (exprimer l'excès), mais il en réactive également le sens littéral. Or si ces verbes expriment l'idée d'absorber une nourriture, cette nourriture non seulement ne nourrit pas, mais elle détruit celui qui ressent un manque. En effet, l'empereur est dévoré par sa haine pour saint Gorgon, qu'il fait cuire, non pas pour le manger, mais par pure vengeance; le cannibalisme de Dioclétien est donc psychologique et symbolique. Ce paradoxe implique que les « nourriture[s] » absorbées n'ont aucune qualité apaisante, et à cet égard manger n'est pas (se) rassasier. En effet, les « ennemis de la religion» «abreuv[ent] » le Christ de « fiel et de vinaigre $^{22} »$, ce qui ne fait qu'augmenter la souffrance, plutôt que l'apaiser. Par ailleurs, la « cruauté [de l'empereur] cherche de nouveaux artifices » pour « vaincre » le martyr, et le sel et le vinaigre qu'il fait appliquer sur les plaies de saint Gorgon « lui éveill[ent] l'appétit ${ }^{23} »$.

\footnotetext{
${ }^{20}$ Bossuet, $1 P d s G$, p. 40.

${ }^{21}$ Bossuet, Second Panégyrique de saint Gorgon, dans Bossuet, Euvres oratoires, 1926, t. 1, p. 583. Nous renverrons désormais à ce discours grâce à l'abréviation $2 P d s G$.

${ }^{22}$ Bossuet, $2 P d s G$, p. 584.

${ }^{23}$ Bossuet, $1 P d s G$, p. 41.
} 
Cette nouvelle férocité est assimilée à un « assaisonnement » qui « rassasi[e $]^{24} »$ la barbarie de l'empereur. Le phénomène de syllepse reconfigure la notion même de satiété. Ainsi, la topique alimentaire n'exprime plus la nécessité d'apaiser un manque (physique ou psychique), mais d'intensifier le mal. Aussi les nourritures évoquées dans les deux Panégyriques sont-elles dépourvues de toute qualité gustative : manger n'est pas savourer. C'est le cas du fiel, du sel et du vinaigre. Il est remarquable qu'entre autres il s'agisse de condiments, de produits qui servent à assaisonner et non pas à nourrir. Ces condiments ne rendent pas le plat plus goûteux, et comme le fiel, ils n'assaisonnent rien d'autre que la douleur du martyr et la haine de Dioclétien.

Le paysage gustatif du récit du martyre actualise donc nettement l'idée de « déplaisir », voire une rhétorique de l'insupportable. La topique alimentaire est franchement mortifère et rance. Ces images semblent éloigner les discours de toute forme de sublime. Cette idée se justifie d'autant plus que, d'un certain point de vue, Longin ne conçoit pas que le sublime puisse s'appuyer sur des motifs liés au « bas corporel ${ }^{25} »$.

Pour autant, alors même qu'il recherche le sublime, Bossuet n'hésite pas à utiliser des images de manducation, qui appartiennent au « bas $\operatorname{corpore}^{26} »$. Or la vulgarité bossuétiste tient aussi en un recours à des images du «bas corporel», de l'abaissement et de l'humiliation physique et physiologique. Dans nos deux Panégyriques de saint Gorgon, le corps humain ne se contente pas d'ingérer, il rejette également. Ainsi en va-t-il du vomissement et de l'excrément. Le vomissement est utilisé comme phénomène d'humiliation diabolique contre les chrétiens et contre tous ceux qui prêchent la foi dans le Christ :

Et quel courage ne fallait-il pas pour exécuter cette généreuse résolution sous Dioclétien, [...] où le diable, sentant approcher peut-être la gloire que Dieu voulait donner à l'Église sous l'empire de Constantin, vomissait tout son venin et toute sa rage contre elle, et faisait ses derniers efforts pour la renverser ${ }^{27}$ ?

Le verbe « vomir » régit deux compléments, l'un concret (« tout son venin »), l'autre abstrait (« toute sa rage »). Le vomissement devient alors un motif d'horreur et de haine contre le saint et contre l'idée même de sainteté. La défécation va dans le même sens :

\footnotetext{
${ }^{24}$ Ibid.

${ }^{25}$ Voir n. 6.

${ }^{26}$ Selon Jean Calvet, Bossuet aime « à éclairer un mot difficile par un quasi-synonyme plus familier, et à définir l'idée abstraite en la ramenant au concret » (Bossuet, 1968, p. 134).

${ }^{27}$ Bossuet, $2 P d s G$, p. 582-583.
} 
Que de peines on prend pour aiguiser un rasoir, que de soins pour l'affiler ! combien de fois le faut-il passer sur la pierre ! Ce n'est, au reste, que pour raser du poil, c'est-à-dire un excrément inutile ${ }^{28}$.

Le motif excrémentiel est lié au superflu, au «danger» et au «pouvoir ${ }^{29}$ » despotique de Dioclétien, et semble s'opposer à l'idée même de vérité que le christianisme entend prêcher en renouvelant la Passion du Christ. Le «bas corporel» bossuétiste est un lieu d'humiliation, d'abaissement, en opposition avec la notion même de sublime. Le corps du martyr devient une pièce de boucherie dont se repaît la cruauté des païens : «On les [les chrétiens] traînait aux gibets tout ainsi qu'on mènerait de pauvres agneaux à la boucherie $[\ldots]^{30} »$. Certes, ces pièces de viande alimentent la barbarie de l'empereur et s'alimentent de celle-ci. Mais en tant que penseur chrétien, Bossuet valorise la souffrance de ceux qui ont donné leur vie pour le Christ, par opposition à ceux qui se complaisent dans l'excès de nourriture, ce qui est le témoignage de leur fanatisme.

\section{Souffrir : la geste mémorable du martyre}

Les jeux de sens que nous avons décrits entraînent une modification du sens du récit du martyre. En effet, il ne s'agit pas seulement de narrer l'horreur de la torture, mais aussi de montrer à nu toute la vérité du dogme chrétien de façon extrêmement spectaculaire. Saint Gorgon est alors mis à l'épreuve de l'« imitation de Jésus-Christ». La structure narrative du Second Panégyrique est réduite à un synopsis du martyre. Pour autant, le récit est construit sur le mode d'un dialogue entre la mort atroce de saint Gorgon et la Passion du Christ : « C'est ainsi qu'il devient conforme à son modèle, qui fait deux plaintes sur les traitements qu'il souffre dans sa Passion ${ }^{31} »$. La plainte du Christ s'appuie sur des motifs de saveur :

[...] et ayant épuisé mes veines de sang, la sécheresse de mes entrailles me causait une soif ardente qui me dévorait la poitrine : voilà le mal qu'ils m'ont fait : Hic plagatus sum; mais lorsque je leur ai demandé à boire avec un grand cri, ils m'ont abreuvé en ma soif de fiel et de vinaigre : Super dolorem vulnerum meorum addiderunt ${ }^{32}$.

\footnotetext{
${ }^{28}$ Ibid., p. 585.

${ }^{29}$ Mary Douglas, De la Souillure, 1992, p. 136. L'auteure ajoute que, comme les marges, les orifices du corps humain sont dangereux, parce qu'ils sont vulnérables. Pour autant, le jugement porté sur les « déchets » produits par le corps varie en fonction des sociétés : tantôt ils sont une source de danger, tantôt ils « sont matière à plaisanterie » (p. 137). Dioclétien ne craint pas de «vomi[r]» $(2 P d s G$, p. 583) sa haine contre saint Gorgon, et à lui « arracher le peu qui lui restait de vie » $(1 P d s G$, p. 42), afin de témoigner de son pouvoir.

${ }^{30}$ Bossuet, $1 P d s G$, p. 38 .

${ }^{31}$ Bossuet, $2 P d s G$, p. 583.

${ }^{32}$ Ibid., p. 583-584. Les deux citations sont respectivement tirées de Zacharie (XIII, 6) et des Psaumes (LXVIII, 31), et signifient «J'ai été percé de ces plaies » et « [...] ils ont ajouté à la douleur de mes plaies des douleurs nouvelles », (La Bible, 1990, p. 1188 et p. 700).
} 
Ici, la dévoration renchérit sur elle-même. En effet, la douleur qui « dévore la poitrine » du saint ne peut être apaisée que par une autre absorption ; or celle-ci n'est pas un remède (comme l'est l'Eucharistie), mais une douleur encore plus intense (« Ils m’ont abreuvé [...] de fiel et de vinaigre »). Par le récit du martyre de saint Gorgon, c'est le modèle du Christ humilié et abaissé qui est réactivé : « [...] et son pauvre corps écorché, à qui les onguents les plus doux, les plus innocents, auraient causé d'insupportables douleurs, est frotté de sel et de vinaigre ${ }^{33} »$. Or par un phénomène d'application et de dialogisation, qui réactive le motif de l'imitation de Jésus-Christ, l'humiliation et l'abaissement cannibales ${ }^{34}$ auxquels est livré saint Gorgon se chargent simultanément d'une forme de grandeur et de sublime : «[...] c'est un sacrifice mystique que la foi nous fait voir sur ces saints autels, et c'est aussi un sacrifice que je dois vous représenter en cette chaire ${ }^{35} »$.

Ce phénomène énonciatif d'application et de dialogisation implique une réflexion sur le sens même des notions de sacrifice et d'exemple. D'une part, on peut considérer que le martyre est une punition, notamment si l'on adopte le point de vue du bourreau. Quand il constate que Gorgon s'est fait chrétien, l'empereur prend une décision violente : « il se résout donc d'en faire un exemple qui pût donner de l'épouvante aux plus déterminés $[\ldots]^{36} »$. Ici, le lexème «punition», associé au syntagme verbal «faire un exemple», renforce l'idée de châtiment. La torture et la mise à mort de celui qui était si apprécié de Dioclétien sont ainsi érigées en menaces destinées à ceux qui trahiraient l'empereur pour rejoindre la secte chrétienne ; la mise à mort et le sang versé ont alors une valeur politique ${ }^{37}$. D'autre part, Bossuet utilise le lexème « exemple » dans son sens moral d'exemplum. Selon le Premier Panégyrique, « les chrétiens ne croyaient point leur [aux martyrs] rendre de plus grands respects qu'en se les proposant pour exemple ${ }^{38} »$. De la même façon, le prédicateur impose le silence à son auditoire en lui rappelant le respect qu'il doit « au récit d'une histoire si mémorable ${ }^{39} »$. Bossuet promet

\footnotetext{
${ }^{33}$ Ibid., p. 583.

${ }^{34}$ « Dès qu' un homme se sert d'un objet, celui-ci devient une partie de lui, et participe de lui. [...] L'appropriation précède l'absorption, elle accompagne la cuisson », (article anonyme « Pur et impur », cité par Mary Douglas, De la Souillure, op. cit., p. 141-142). En faisant cuire et en assaisonnant saint Gorgon, le but de Dioclétien était d'humilier son ancien favori en faisant pénétrer dans son corps des substances (le fiel et le vinaigre) qui ne lui appartiennent pas. La cuisson du corps humain avait pour but de faire perdre toute qualité humaine à saint Gorgon.

${ }^{35}$ Bossuet, $2 P d s G$, p. 577.

${ }^{36}$ Bossuet, $1 P d s G$, p. 40 ; c'est moi qui souligne.

${ }^{37}$ «Les rites agissent sur le corps politique par le moyen terme symbolique du corps physique », écrit Mary Douglas (De la Souillure, op. cit., p. 143).

${ }^{38}$ Bossuet, $1 P d s G$, p. 33.

${ }^{39}$ Ibid.
} 
une narration qu'il fera « simplement et brièvement ${ }^{40} »$. Ainsi, le prédicateur revendique un art de la narration dont le but illocutoire sera moral et édifiant.

Par là même, la mort par le martyre devient un moment de témoignage apporté par le saint au dogme chrétien. Précédée par une série d'abaissements et d'humiliations, la mort modifie la valeur de la topique alimentaire. Elle est alors représentée comme un instant de joie «Voilà, Messieurs, quelle a été la fin de notre martyr, qui a méprisé le monde dans ses promesses et dans ses menaces, dans ses délices et dans ses tourments ; $[\ldots]\rangle^{41}$. Ici, le lexème « délices » revêt une acception psychique, mais aussi une acception physique, gustative. La joie de saint Gorgon devient alors la joie du chrétien qui rejoint son créateur après avoir rendu témoignage de sa foi. Mais c'est surtout l'élévation de l'âme des auditeurs qui est ici en jeu, dans la mesure où l'hésitation entre le récit du martyre et celui d'un sacrifice animal entraîne le renversement paradigmatique de l'exécution barbare en célébration chrétienne. Aussi ce renversement fait-il advenir de façon paradoxale le sublime bossuétiste.

\section{Témoigner : l'élévation dans l'abaissement}

Si l'on a pu parler de «mauvais goût » pour caractériser le style de Bossuet dans le Second Panégyrique de saint Gorgon ${ }^{42}$, il convient de nuancer ce jugement - qui est d'ordre moral, bien plus qu'esthétique et poétique -, et de considérer que la topique alimentaire et celle $\mathrm{du}$ « bas corporel » se donnent comme une reconfiguration de la liturgie catholique, notamment de l'Eucharistie et de la transsubstantiation. Cette élévation paradoxale se veut « un ordre secret de la Providence », que la poétique du panégyrique a pour fonction de mettre en récit. Certes, le corps du saint est rapproché d'une pièce de viande que le «tyran » fait cuire afin d'humilier et d'abaisser les chrétiens. Cela étant, il ne serait pas non plus improbable que ce passage du Premier Panégyrique vienne confirmer l'existence de deux topö̈, à ce jour déjà présents dans le Thésaurus. En effet, il peut s'inscrire dans le topos «Tyran_inciter_action_cruelle» ou «Un tyran oblige ses sujets à des actes cruels » dans sa formulation complète ${ }^{43}$, dans la mesure où Dioclétien « fait coucher le saint martyr sur un gril de fer déjà tout rouge par la véhémence de la chaleur ${ }^{44} »$. Le passage de Bossuet peut également illustrer le topos

\footnotetext{
${ }^{40}$ Ibid., p. 33-34.

${ }^{41} \mathrm{Ibid} .$, p. 42.

42 Jean Calvet écrit: «Bossuet n'a pas été tenté, ou il n'a été tenté qu'un moment, par le style précieux; sa vigoureuse nature répugnait à la mièvrerie. Mais il a aimé la rhétorique bruyante ornée de verroteries et d'un certain réalisme dont le mauvais goût est fait surtout de naïveté » (Bossuet, op. cit., p. 129-130). Jean Calvet fonde notamment son analyse sur le Second Panégyrique de saint Gorgon.

${ }^{43} \mathrm{https}: / /$ www.satorbase.org/index.php?do=topoi\&topoi=10740 ; page consultée le 24/06/2019.

${ }^{44}$ Bossuet, $1 P d s G$, p. 41.
} 
« cannibalisme_par_vengeance»(«Un personnage commet ou fait commettre un acte de cannibalisme par vengeance » dans sa formulation complète ${ }^{45}$ ), dans la mesure où l'empereur se venge sur saint Gorgon (et par métonymie, sur les chrétiens), qu'il avait en grâce, de ce que qu'il considère comme une trahison.

Mais Bossuet lui-même donne à ses auditeurs les clés qui permettent de lire et de comprendre cet événement humiliant au regard de la foi et des Écritures, et qui n'est qu'une trahison aux yeux de l'empereur :

Ces âmes héroïques n'ont pu plaire au monde, et le monde ne leur a pu plaire : voilà la cause de leurs contrariétés. Le monde ne leur a pas plu, c'est pourquoi ils l'ont méprisé. Ils n'ont pas plu au monde, de là vient que le monde a pris plaisir d'affliger ce qui n'était pas à lui. Et le tout est arrivé par un ordre secret de la Providence, afin d'accomplir cette parole mémorable de notre divin Sauveur : « Je ne suis pas venu pour donner la paix, mais pour allumer la guerre Non veni pacem mittere, sed gladium ${ }^{46}$.

Par un phénomène de dialogisme interdiscursif, Bossuet donne la légende, c'est-à-dire, le mode de fonctionnement du sublime qu'il entend mettre en place. Aussi est-ce sur le dogme chrétien que cette forme de sublime s'appuie. Un « ordre secret de la Providence » émerge de la haine réciproque que se vouent les «défenseurs de la foi ${ }^{47}$ » et les ennemis du Christ et de l'impitoyable combat qu'ils se livrent, qui explique les contradictions, les «contrariétés» inhérentes à la sainteté elle-même et au salut. Ni le Christ ni le martyr ne cherchent alors à résoudre ces contradictions, mais au contraire à les maintenir, en vertu d'une indéfectible volonté de «dire la véritét8 »: «Ah! Messieurs, humilions-nous sous la puissante main de Dieu, de peur qu'après avoir tout perdu, nous ne perdions encore l'affliction que nos ruines nous apportent, au lieu de la faire profiter à notre salut ${ }^{49}$ ». La tension à la fois abaissante et glorificatrice qui structure le sublime chrétien actualise d'un point de vue rhétorique un des fondements du dogme selon lequel Jésus-Christ est « un signe de contradiction ${ }^{50} »$. En définitive, dans tout ce qu'elle comporte de violent et de glauque, la topique du manger et du boire renvoie l'auditeur à sa propre condition, à sa propre âme et surtout à son propre corps.

La mort du martyr n'est donc pas une fin en soi - du moins, elle ne l'est que pour le «tyran » qui l'a condamné. Aussi est-ce toute la représentation du corps humain qui est

\footnotetext{
${ }^{45}$ https://www.satorbase.org/index.php?do=topoi\&topoi=10226 ; page consultée le 24/06/2019.

${ }^{46}$ Bossuet, $1 P d s G$, p. 35. La citation latine vient de Matthieu $(\mathrm{X}, 34)$ et signifie « [...] je ne suis pas venu y [sur la terre] apporter la paix, mais l'épée » (La Bible, op. cit., p. 1279).

${ }^{47}$ Ibid.

${ }^{48}$ Ibid., p. 32.

${ }^{49}$ Ibid., p. 45

${ }^{50}$ Ibid., p. 38.
} 
reconfigurée. Selon l'axiologie chrétienne, la condamnation, l'humiliation, la destruction du corps dans la mort ne sont que le prélude à un renouvellement de toutes les choses mortelles et terrestres : « Or il en est des martyrs comme d'un excellent original dont chaque peintre cherche de copier quelques traits pour embellir son ouvrage ${ }^{51} »$. La mort abaissante est assimilée à un travail, à la fois celui du peintre (dans un régime métaphorique) et celui du bourreau (si l'on envisage le sens étymologique du terme, «torture »). L'endurance et la constance du saint devant les puissances de la mort deviennent un sujet d'étonnement et d'admiration, et un appui solide au sublime chrétien, dans tout ce qu'il a de paradoxal : «Vous avez vu en esprit comme la constance de Gorgon a duré jusqu'à la mort, dont il a goûté à longs traits toute l'amertume ; $[\ldots]^{52} »$. En associant un sens physiologique (celui du goût) et un sens moral, la syllepse qui porte sur le lexème « amertume » montre que Bossuet se refuse à établir un démarcage absolu entre le corps et les sensations d'un côté, et l'esprit et l'activité de l'intelligence de l'autre côté. Il est donc possible de voir en esprit, comme si la raison pouvait avoir les mêmes fonctions que les yeux.

En définitive, le discours chrétien requalifie la nourriture, qu'elle soit en excédent ou en défaut, pour en faire le mode de compréhension de « la source du mal ${ }^{53}$ ». Selon Bossuet, cette « source » est la disproportion qui préside à la répartition des richesses et des nourritures terrestres : «le riche prépare ses greniers pour engloutir la nourriture du pauvre », qui demeure « en son extrême indigence ${ }^{54}$ ». L'humiliation du corps du saint considéré comme nourriture devient alors le signe de «l'éminente dignité des pauvres dans l'Église ». C'est le Christ luimême qui a institué cette dignité, lorsqu'il « est venu au monde pour renverser l'ordre que l'orgueil y a établi $[\ldots]^{55}$ ». Dans les récits de la mort de saint Gorgon, c'est la topique alimentaire qui structure sur le plan énonciatif ce renversement. À travers la topique alimentaire, la nourriture devient alors elle-même un langage, celui du Christ abaissé, puis relevé.

\section{Les topoi du manger et du boire : de la topique à la légende}

Les analyses que nous avons menées nous paraissent pouvoir contribuer à l'étude d'un cas-limite de fiction narrative, à savoir le récit de martyre, tel qu'il est construit par Bossuet

\footnotetext{
${ }^{51}$ Ibid., p. 43.

${ }^{52}$ Ibid., p. 42.

${ }^{53}$ Ibid., p. 45.

${ }^{54}$ Ibid.

${ }^{55}$ Bossuet, Sermon sur l'éminente dignité des pauvres dans l'Église, dans Euvres oratoires, 1927, t. 3, p. 121.
} 
dans ses deux Panégyriques de saint Gorgon. Aussi les outils d'analyse du discours ont-ils vocation à venir enrichir les techniques d'analyse des topoï, que la SATOR a à cœur d'élaborer et d'utiliser. Et c'est pourquoi nous ne souhaitons pas ici formuler à proprement parler une conclusion de cet article, mais plutôt dresser une sorte de bilan, portant à la fois sur la méthodologie d'analyse topique dans ses rapports avec d'autres champs disciplinaires, et sur les motifs du manger et du boire chez Bossuet.

Le panégyrique utilise le matériau hagiographique, et actualise le matériau légendaire à un niveau oratoire et sublime. André Jolles définit la légende comme une forme simple. Les formes simples comme des formes «qui, tout en procédant également du langage, ne comportent pas [...] cette consolidation ${ }^{56} »$ que permet le langage; les formes simples « procèdent d'un travail du langage lui-même, sans intervention, pour ainsi dire, d'un poète ${ }^{57}$ ». C'est pourquoi les formes simples sont structurées par des «dispositions mentales» spécifiques. La disposition mentale de la légende est celle de l'imitation ${ }^{58}$.

À bien des égards, voir en esprit, c'est à la fois imaginer et se laisser convaincre. C'est pourquoi le prédicateur impose à l'auditeur de se conformer au modèle du saint :

[...] reste maintenant que vous imitiez sa foi, cette foi ardente qui lui a fait préférer à tous les honneurs l'opprobre de Jésus-Christ, et a tenu son esprit entier et inébranlable, pendant que son corps s'en allait pièce à pièce comme une vieille masure ${ }^{59}$.

Le corps et l'esprit du saint agissent l'un par rapport à l'autre comme une balance à plateaux : si l'un est abaissé, l'autre est élevé ${ }^{60}$. C'est ce double mouvement conjoint qui caractérise le plus exactement le sublime chrétien. L'élévation de l'âme vers Dieu devient alors consubstantielle à l'humiliation du corps, parce que toute matière est éphémère et consommable. C'est donc cette dialectique qui justifie l'utilisation et la modulation de la topique alimentaire. Par ailleurs, c'est également à ce double mouvement que Bossuet invite l'auditeur, par un phénomène, non plus de compensation, mais d'analogie, en dehors duquel le chrétien est en état de péché. L'analogie fonde donc la conception bossuétiste de la pénitence. Une telle configuration narrative est propre à illustrer le topos, présent dans Satorbase, «révolte_contre_tyran_cruel», ou «Le peuple se révolte contre la tyrannie ${ }^{61} »$ dans sa

\footnotetext{
${ }^{56}$ André Jolles, Formes simples, op. cit., p. 17.

${ }^{57}$ Ibid., p. 18.

${ }^{58}$ Ibid., p. 36.

${ }^{59}$ Bossuet, $1 P d s G$, p. 42.

${ }^{60} \mathrm{Au}$ sens propre comme au sens métaphorique des verbes abaisser et élever.

${ }^{61} \mathrm{https}$ ://www.satorbase.org/index.php?do=topoi\&topoi=10605 ; consulté le 24/06/2019.
} 
formulation complète. En effet, l'imitation de saint Gorgon, à laquelle le prédicateur incite les fidèles, peut se lire, selon l'axiologie chrétienne, comme une forme de révolte et de résistance contre les ennemis de la foi et de l'Église, qui menacent son intégrité. S'il est vrai qu'à l'époque où il prononce les Panégyriques de saint Gorgon, Bossuet ne s'est pas encore engagé dans la mission qui consistera à ramener les réformés dans le giron de l'Église, il n'en demeure pas moins que, d'une certaine manière, les discours de jeunesse que nous étudions ici témoignent d'un souci d'unité des chrétiens, d'une part devant la toute-puissance de Dieu, d'autre part face à tout ce qui menace l'institution et le dogme chrétiens.

Le prédicateur doit donner une première impulsion à ce double mouvement, et la maintenir, en vue du salut des hommes et du triomphe de la vérité. À cet égard, les motifs du manger et du boire dans les Panégyriques de saint Gorgon revêtent une valeur émotionnelle forte : «Que si nous sommes assez généreux pour dédaigner ses faveurs [celles du Christ], il nous représente un grand appareil de peines et de supplices pour nous émouvoir ; $[\ldots]^{62} \gg$. Les lexèmes «peines» et «supplices» se donnent ici comme des termes génériques à partir desquels le récit du martyre comme rite cannibale va se développer. Aussi le but illocutoire du récit de la mort du saint est-il clair : il faut «émouvoir». Or le movere ne suffit pas, encore doit-il être associé au docere, encore faut-il « instruire ». Tel un animal sacrifié, saint Gorgon verse son sang pour le Christ. Bossuet ajoute « [...] il faut que ce sang échauffe le nôtre ${ }^{63} »$. La tournure impersonnelle « il faut » actualise ici la fonction déontique du langage. En sortant de la bouche du prédicateur, la parole s'offre alors en discours d'autorité qui nourrit le cœur et l'âme de l'auditeur. La chronographie retenue par l'orateur permet de rapprocher le temps de la mort du saint, et le hic et nunc du discours prononcé : « il n'est point de temps ni d'heure plus propre à faire l'éloge des saints martyrs, que celui du sacrifice adorable pour lequel vous êtes ici assemblés ${ }^{64} »$. En écoutant le discours du garant de l'autorité ecclésiastique, les auditeurs se nourrissent d'une « nourriture céleste ${ }^{65}$ » qui élève l'âme vers Dieu. La fonction de la parole sacrée prononcée en chaire relève alors du sublime : la « joie ${ }^{66} »$ n'est pas dans le monde, elle est auprès de Dieu.

Or le témoignage apporté par le saint ne doit pas être un événement digne d'oubli. En effet, la mort abaissante doit se changer en souvenir glorieux et édifiant. Aussi est-ce cette

\footnotetext{
${ }^{62}$ Bossuet, $1 P d s G$, p. 34.

${ }^{63}$ Bossuet, $2 P d s G$, p. 579.

${ }^{64}$ Ibid., p. 576.

${ }^{65}$ Ibid.

${ }^{66}$ Longin, Traité du sublime, op. cit., p. 81.
} 
transformation qui va faire advenir le sublime bossuétiste dans toute sa singularité. Les motifs du manger et du boire entraînent l'utilisation d'une autre topique qui leur est conjointe, celle des odeurs. Saint Gorgon sur le « gril de fer déjà rouge par la véhémence de la chaleur ${ }^{67}$ » est décrit comme une pièce de viande : Gorgon « fon[d] de tous côtés par la force du feu ${ }^{68} »$. « [À] l'entour de lui » s'élève « une vapeur noire que le tyran humait pour contenter son avidité69 ». Bossuet évoque enfin le «corps rôti ${ }^{70} »$ du saint. Mais le motif de l'odeur permet de faire basculer cette évocation de «mauvais goût ${ }^{71}$ » (sans mauvais jeu de mots !) dans l'ordre du sacré et du sublime : « Saint Gorgon, en mourant, a laissé une certaine odeur de sainteté sur la terre $^{72} \gg$, dit M. de Meaux dans l'exorde du Premier Panégyrique. Le récit sordide du martyre est ici transfiguré en symbole d'élévation et de pureté. Dans le Second Panégyrique, le rite barbare et cannibale ${ }^{73}$ dont saint Gorgon a été la victime bascule dans l'ordre du sacré : «Les prières qu'il faisait monter au ciel changeaient cette fumée noire en encens $[\ldots]^{74} »$. L'encens est un parfum répandu lors de la messe, à l'image de l'« odeur de suavité » en laquelle JésusChrist « s'est offert pour nous ${ }^{75}$ ». L'odeur, la fumée ont une fonction mémorielle et sacrificielle forte. En effet, saint Gorgon est mené au sacrifice comme une pièce de viande ${ }^{76}$. Il est cuit de la même façon qu'on cuirait des agneaux, et son corps devient «aussi vil que celui des $\operatorname{animaux}^{77} »$.

Cette édification paradoxale que Bossuet propose à ses auditeurs s'appuie sur un ensemble de « gestes verbaux », constitutifs de la forme de la légende. Les gestes verbaux sont du langage, impensable au niveau conceptuel. Dans les Panégyriques de saint Gorgon, les

\footnotetext{
${ }^{67}$ Bossuet, $1 P d s G$, p. 41.

${ }^{68}$ Ibid.

${ }^{69}$ Ibid., p. 42.

${ }^{70}$ Bossuet, $2 P d s G$, p. 584.

71 Voir note 42.

${ }^{72}$ Bossuet, $1 P d s G$, p. 33.

${ }^{73}$ Dans la littérature narrative de l'époque baroque, le cannibalisme est une source d'inspiration et un ressort narratif que les auteurs d'histoires tragiques exploitent largement. On pense, par exemple, aux « Entre-mangeurs » et au « Cœur mangé », deux récits publiés en 1630 dans les Spectacles d'horreur de Jean-Pierre Camus (Christian Biet (dir.), Théâtre de la cruauté et récits sanglants en France (XVI -XVII siècle), 2006, p. 273-274 et p. 259 263), à l'Histoire prodigieuse d'une jeune demoiselle laquelle fit manger le foie de son enfant à un jeune gentilhomme, publiée en 1608 (ibid., p. 494-501), au récit «D’Astober gentilhomme de Tuderte, dans le duché de Spolète, qui fut mangé par une populace enragée », publié en 1622 dans Le Théâtre tragique de Pierre Boitel (ibid., p. 331). De façon assez singulière, ces histoires tragiques et les Panégyriques de saint Gorgon semblent entrer en résonnance, et témoignent - tantôt dans une veine profane, tantôt dans une veine chrétienne - de la fascination qu'exerce le cannibalisme sur l'imaginaire au XVII ${ }^{\mathrm{e}}$ siècle. (Voir l'article de M. Moser, «Le cœur mangé à plusieurs sauces $\gg$. dans ce volume.)

${ }^{74}$ Bossuet, $2 P d s G$, p. 584.

${ }^{75}$ Bossuet, $1 P d s G$, p. 46.

${ }^{76}$ Ibid., p. 38.

${ }^{77}$ Bossuet, $2 P d s G$, p. 582.
}

Topiques, Études satoriennes, 5, 2020, Le Boire et le manger, https://journals.uvic.ca/index.php/sator/index 
gestes verbaux sont la mise sur le gril du saint, sa cuisson, son assaisonnement, les odeurs qu'il exhale, sa mort sacrificielle ; et de fait, au-delà même du dégoût que suscite l'évocation du martyre de saint Gorgon, l'assimilation d'un être humain à une pièce de boucherie présente un aspect incongru, mais défie également les facultés d'intellection des auditeurs. Bien que Jolles refuse de désigner les gestes verbaux comme des $« \operatorname{motifs}^{78} »-$ la désignation de topos ne lui conviendrait pas plus -, les gestes verbaux sont ici construits par la topique alimentaire. Ainsi, dans la mesure où le matériau est ici légendaire, l'assimilation de saint Gorgon à un animal mené au sacrifice perd sa valeur métaphorique, et devient pleinement topique. Pour autant, c'est le caractère à la fois évocateur et inintelligible des gestes verbaux qui crée la disposition mentale d'imitation. Ces «gestes verbaux» d'innutrition sont la représentation d'un «concept unique $^{79} »$, qui structure la disposition mentale d'imitation et le but illocutoire d'édification.

En définitive, la forme du panégyrique se donne comme la « [f]orme simple actualisée ${ }^{80} »$ de cette forme simple qu'est la légende, en ceci que, dans le panégyrique, les gestes verbaux sont disposés «de telle sorte qu'ils peuvent à tout instant être orientés et reliés d'une manière déterminée pour atteindre ainsi une importance actuelle ${ }^{81} \gg$. C'est ainsi que Bossuet édifie ses auditeurs par l'exemple de saint Gorgon dévoré. Par le geste verbal de la cuisson et de la manducation du saint - construction purement verbale et inintelligible -, le prédicateur invite à réfléchir sur la consommation des biens offerts aux hommes par Dieu : «[...] si nous voulons qu'il nous fasse miséricorde, ayons compassion de nos pauvres frères, que la misère du temps réduira peut-être à d'étranges extrémités ${ }^{82}$ ». Par un phénomène de dialogisme et d'application de la légende de saint Gorgon au monde contemporain, l'Aigle de Meaux met ici en garde les fidèles contre le mésusage des biens et des nourritures terrestres qui menace de faire des chrétiens des dévorateurs excessifs et sacrilèges - à faire des chrétiens autant d'autres Dioclétiens. La péroraison du Premier Panégyrique est aussi un plaidoyer en faveur d'une abondance qui soit également prospérité pour les peuples :

Ainsi puissions-nous recevoir abondamment les faveurs du ciel ; que Dieu rende le premier lustre à cette ville autrefois si fleurissante ; qu'il rétablisse les campagnes désolées ; qu'il fasse revivre partout aux environs le repos et la douceur d'une paix bien affermie $;[\ldots]^{83}$

\footnotetext{
${ }^{78}$ André Jolles, Formes simples, op. cit., p. 42.

${ }^{79}$ Ibid., p. 41.

${ }^{80}$ Ibid., p. 43.

${ }^{81}$ Ibid.

${ }^{82}$ Bossuet, $1 P d s G$, p. 46.

${ }^{83}$ Ibid.
} 
Ce passage est une prière pour la ville de Metz, qui est, à l'époque, dans une situation politique et sociale très instable ${ }^{84}$. Le style de cette prière s'appuie sur une forme de lyrisme et de sublime, ainsi qu'en témoignent les séquences exclamatives selon le patron syntaxique que + sujet + $V_{\text {impératif }}+$ compl., les adverbes intensifs de sens hyperbolique (« abondamment», « si ») et un vocabulaire axiologiquement euphorique («faveurs », "premier lustre», « rétablisse», « repose», «douceur»). Les motifs du manger, du boire, de la manducation et de la consommation n'ont plus seulement un statut de topö̈, mais aussi de gestes verbaux ; c'est cette double fonction des motifs du manger et du boire qui stabilise le but édifiant et argumentatif de la rhétorique du sublime. À l'instar de la nourriture, le matériau hagiographique a été mangé, digéré et transformé en une substance qui nourrit l'édification et le sublime chrétiens. Par là même, le texte bossuétiste est « scriptible », bien plus qu'il est « lisible ${ }^{85} »$.

\section{Conclusion}

Bien que Longin lie au sublime tout discours épidictique (« ...] les panégyriques et tous ces discours qui ne se font que pour l'ostentation ont partout du Grand et du Sublime ; $\left.[\ldots]^{86} \gg\right)$, les deux discours que nous venons d'étudier sont a priori éloignés de toute forme de sublime et d'élévation, tant la topique alimentaire y est utilisée de façon dysphorique. Ceux-ci servent une rhétorique propre à susciter la pitié devant le terrible spectacle du martyre. Pour autant, le propre du sublime chrétien est d'être en proie à la contradiction et au renversement, et d'articuler la condamnation des plaisirs de la chair et la promesse des délices du monde spirituel. En effet, l'élévation se trouve dans l'abaissement lui-même. D'un point de vue à la fois topique et énonciatif, les motifs du manger et du boire connaissent plusieurs recatégorisations, et se donnent comme les outils linguistiques de la glorification et de la mémoire du saint, ainsi que de l'instruction des fidèles. Non seulement les topoï du manger, du boire et de la consommation sont recatégorisés, mais ils sont eux-mêmes mâchés, digérés, transformés et assimilés à la poétique même du panégyrique, de son but édifiant et sublime. Or si le sublime chrétien semble paradoxal, il correspond plutôt à un point du dogme selon lequel

\footnotetext{
${ }^{84}$ Sur ce sujet, voir Georges Minois, Bossuet. Entre Dieu et le soleil, op. cit., p. 76-77.

${ }^{85}$ Barthes distingue les «textes lisibles » $(S / Z, 1970$, p. 11), lus « dans une sorte d'oisiveté, d'intransitivité, [...] de sérieux » (ibid., p. 10), et les «textes scriptibles» (ibid., p. 10), ouverts à «la pluralité des entrées, [à] l'ouverture des réseaux, [à] l'infini des langages » (ibid., p. 11). La manducation et la digestion de la topique alimentaire, ainsi que les effets de dialogisme, permettent d'envisager les discours de Bossuet comme «textes scriptibles ».

${ }^{86}$ Longin, Traité du sublime, op. cit., p. 83.
} 
«les derniers seront les premiers et les premiers seront les derniers ${ }^{87}{ }$ (Matthieu, XX, 16). En définitive, il ne peut y avoir de sublime au sens où l'entend Longin qu'autant qu'il y a subversion de Longin. Fondée sur la topique alimentaire, la rhétorique du sublime devient une rhétorique du déplaisir, par laquelle Bossuet joint l'utile ... au désagréable.

\section{Bibliographie}

\section{Euvres et sources}

La Bible, Paris, 1990, Robert Laffont (Bouquins) [Ph. Sellier (eds.) / L.-I. Lemaître de Sacy (trad.)].

BOSSUET, Jacques Bénigne, Premier Panégyrique de saint Gorgon, dans Euvres oratoires de Bossuet, Bruges-Paris, Desclée-De Brouwer et Cie, 1926, vol. 1, p. 31-46 [J. Lebarcq, Ch. Urbain et E. Levesque (eds.)].

BOSSUET, Jacques Bénigne, Second Panégyrique de saint Gorgon, dans Euvres oratoires de Bossuet, Bruges-Paris, Desclée-De Brouwer et Cie, 1926, vol. 1, p. 576-586 [J. Lebarcq, Ch. Urbain et E. Levesque (eds.)].

BOSSUET, Jacques Bénigne, Sermon sur l'éminente dignité des pauvres dans l'Église, dans Euvres oratoires de Bossuet, Bruges-Paris, Desclée-De Brouwer et Cie, 1927, vol. 3, p. 119-138 [J. Lebarcq, Ch. Urbain et E. Levesque (eds.)].

BOSSUET, Jacques Bénigne, Pensées chrétiennes et morales, dans Euvres oratoires de Bossuet, Bruges-Paris, Desclée-De Brouwer et Cie, 1926, vol. 6, p. 640-707 [J. Lebarcq, Ch. Urbain et E. Levesque (eds.)].

LONGIN, Traité du sublime, Paris, Librairie Générale Française, 1995 [F. Goyet (eds.) / Boileau (trad.)]

\footnotetext{
${ }^{87}$ Bossuet cite cette phrase de l'Évangile de Matthieu dans le Sermon sur l'éminente dignité des pauvres dans l'Église ; op. cit., p. 121. 
VORAGINE, Jacques de, La Légende dorée, Paris, Garnier-Flammarion, 1967, t. 2 [Révérend Père H. Savon (eds.) / J.-B. M. Roze (trad.)].

\section{Études}

BARTHES, Roland, S/Z, Paris, Seuil (Points), 1970.

BAKHTINE, Mikhaïl, L'Euvre de François Rabelais et la culture populaire au Moyen Âge et sous la Renaissance, Paris, Gallimard (Tel), 1970 [A. Robel (trad.)].

BIET, Christian (dir.), Théâtre de la cruauté et récits sanglants en France (XVI ${ }^{e}-X V I I^{e}$ siècle), Paris, Robert Laffont, (Bouquins), 2006.

CALVET, Jean, Bossuet, Paris, Hatier (Connaissance des lettres), 1968.

DOUGLAS, Mary, De la Souillure. Essai sur les notions de pollution et de tabou, Paris, La Découverte (Poche), 2005 [A. Guérin (trad.)].

JOLLES, André, Formes simples, Paris, Seuil (Poétique), 1972 [A. M. Buguet (trad.)].

MINOIS, Georges, Bossuet. Entre Dieu et le Soleil, Paris, Perrin, 2003.

WEILL, Michèle, RODRIGUEZ, Pierre, « Objectifs littéraires de la Sator », https://satorbase.org/index.php?do=outils\#objectifs, page consultée le 12/05/19. 